\title{
Revision on the Recent Diagnostic Strategies of Fungal Infections
}

\author{
Amina Mostafa Abd El-Aal ${ }^{1}$, Noha El-Mashad ${ }^{*}$, Al-Shaymaa Nasr Mohamed ${ }^{2}$ \\ ${ }^{1}$ Department of Clinical Pathology, Faculty of Medicine, Mansoura University, Mansoura, Egypt \\ ${ }^{2}$ Department of Clinical Pathology, Aga Central Hospital, Mansoura, Egypt \\ Email:^nmashad@hotmail.com, agomah@hotmail.com
}

How to cite this paper: El-Aal, A.M.A., El-Mashad, N. and Mohamed, A.-S.N. (2017) Revision on the Recent Diagnostic Strategies of Fungal Infections. Open Journal of Medical Microbiology, 7, 29-40. https://doi.org/10.4236/ojmm.2017.71003

Received: February 10, 2017

Accepted: March 11, 2017

Published: March 14, 2017

Copyright (c) 2017 by authors and Scientific Research Publishing Inc. This work is licensed under the Creative Commons Attribution International License (CC BY 4.0).

http://creativecommons.org/licenses/by/4.0/

\section{Open Access}

\begin{abstract}
Many invasive fungal diseases have no characteristic signs or symptoms, and may give negative blood cultures; therefore, clinician may decide to start empirical antifungal therapy particularly in high risk patient (e.g., hematopoietic stem cell transplant, solid organ transplant, and AIDS patients). This increases the incidence of appearance of resistant fungal strains to antifungal drugs. Traditional methods for diagnosis such as wet mount examination and microbiological cultures remain the gold standard methods for fungal disease diagnosis. However, they are time-consuming, insensitive, and have a limited impact on clinical decision-making. Significant progress has recently achieved in diagnostic tools of fungal disease. Antigen and antibody based assays, molecular techniques, and MALDI TOF spectrometry technique and nanotechnology offer more rapid, sensitive and accurate results.
\end{abstract}

\section{Keywords}

Fungal Infections, Conventional Diagnostic Techniques, Mannan, MALDI-TOF MS, Molecular Diagnostic Techniques

\section{Review}

Since 1960, sudden and extreme rise in fungal infections was observed. They currently represent a global health threat [1]. Diseases caused by fungi are called mycoses, and classified to superficial, subcutaneous, and systemic mycoses. Superficial mycoses affect skin, mucosal membranes, hair, and nails. Dermatophytosis, superficial candidiasis, tineaversicolor, and piedra are examples of superficial mycoses (Figure 1). Subcutaneous mycoses involve dermis of the skin, subcutaneous tissues, muscle and fascia (Figure 2). Systemic mycoses affect internal organs and deep tissues of the body. They are such as histoplasmosis, invasive aspergillosis, invasive candidiasis, and cryptococcosis (Figure 3) [2] [3]. 

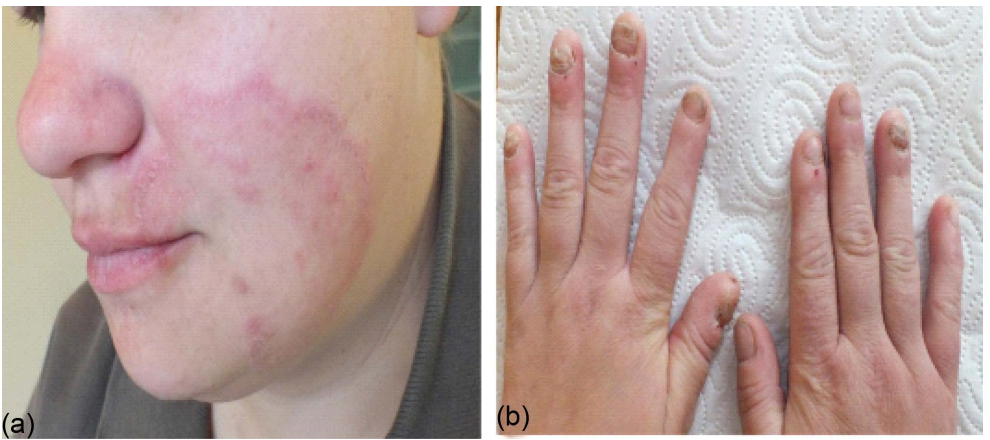

Figure 1. Superficial mycoses in a 34 -year-old woman. (a) Tinea faciei caused by Trichophyton rubrum. (b) Tinea unguium and tinea manuum caused by Trichophyton rubrum in the same patient [4].

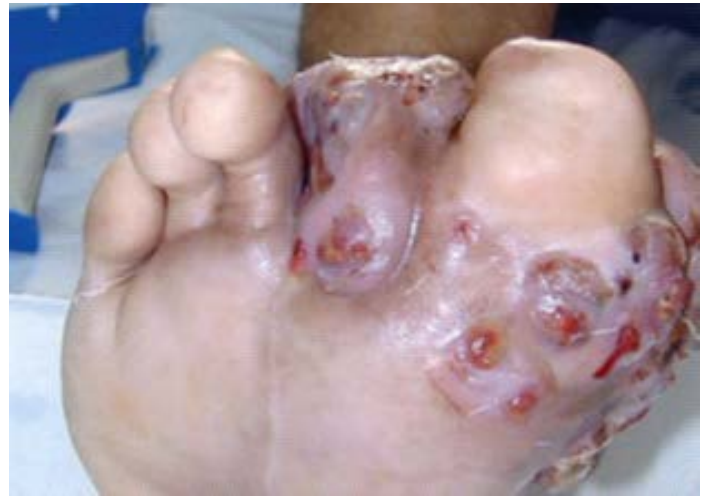

Figure 2. Subcutaneous mycosis, Eumycetoma due to Madurella mycetomatis showing multiple sinus tracts [5].

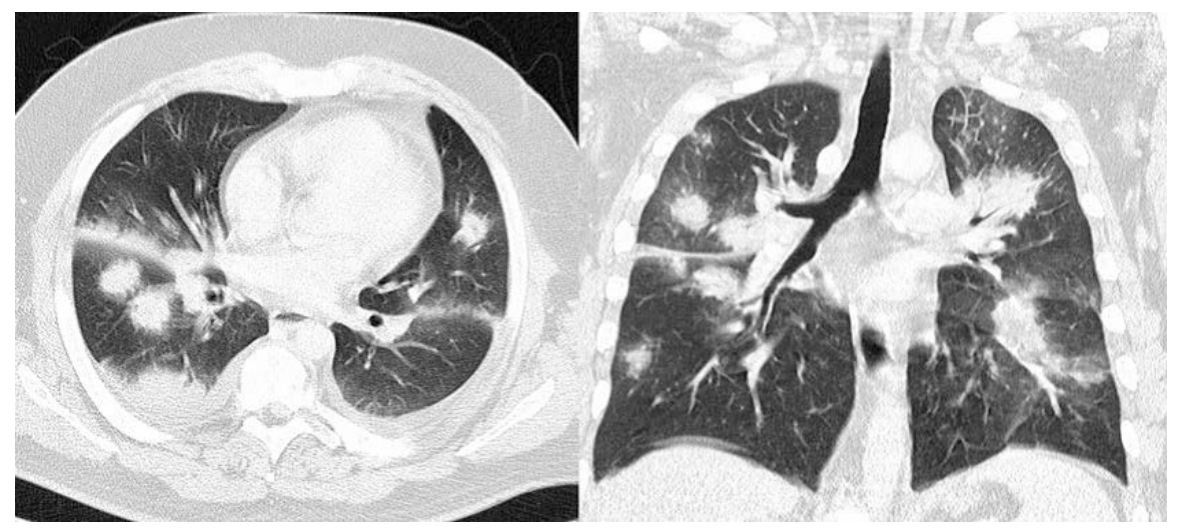

Figure 3. Angioinvasive aspergillosis in a 35-year-old man with acute lymphatic leukemia. CT scan shows bilateral peribronchovascular nodular lung lesions with surrounding halo of ground-glass attenuation representing adjacent hemorrhage [6].

Accurate identification of the causative agent is essential due to the increased use of prophylactic schedules that predispose the patient to the infection by fungal species which are intrinsically resistant to the available antifungal agents e.g., Candida glabrata and Candida krusei [7].

Large volumes of specimens are needed in fungal disease diagnosis due to lower amount of viable elements. The most common specimens are hairs, skin, 
nails, blood, bone marrow, CSF, and respiratory secretions. The collected specimens must be placed in a suitable sterile container, and sent to the laboratory without delay. Sterile specimens and those that are suspected to contain dermatophytes do not need refrigerator [8].

Conventional diagnostic techniques used for fungal infections, such as direct microscopic examination of clinical specimens and microbial cultures, remain the gold standard methods for fungal pathogens detection and identification. However, they are less sensitive and/or specific. Most pathogenic fungi are considered as risk group 2 pathogens, so, biological safety level recommendations (BSL) should be followed during plating and identification using biological safety cabinet class II [9].

\section{Direct microscopic examination}

Direct examination of clinical material can provide rapid report to physician, and allows early initiation of appropriate treatment. Budding yeasts with pseudohyphae suspect Candida species, while hyphae form with arthrospores which are sometimes seen in skin, nail scrapings, and hair can suspect dermatophytes. Hyphae with cross-septa found in sputum or BAL specimen suspect Aspergillus [10].

Most specimens can be examined using $10 \%-20 \% \mathrm{KOH}$ that digests keratin in fungal cell wall (Figure 4). Addition of calcofluor white and subsequent examination by fluorescence microscopy enhances the detection of most Fungi. Gram staining may also be useful for diagnosis of yeast infections as mucocutenous candidiasis (Figure 5). Giemsa staining of smears is recommended for detection of yeast cells of Histoplasma capsulatum (Figure 6). India ink preparations can be used to detect encapsulated yeast cells of Cryptococcus in CSF specimens (Figure 7) [11].

Various microscopic techniques are used in diagnosis of fungal infections. These techniques involved use of classical light microscopes and electron microscopy. However, advancement of microscopic techniques such as fluorescent microcopy, confocal laser scanning microscopy, atomic force microscopy (AFM) and others involving integration of automated computer monitoring have aided significantly to the mycological research. The AFM has been used in viewing and analyzing the ultra-structure of fungal cell surface and analyzing structure of native membrane proteins at sub-nanometer resolution [16].

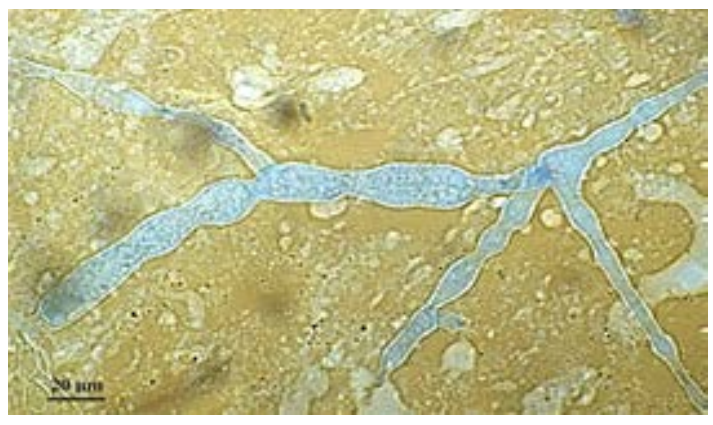

Figure 4. Direct mount in potassium hydroxide of aspirated material from a case of subcutaneous mucormycosis showing thin-walled hyphae [13]. 


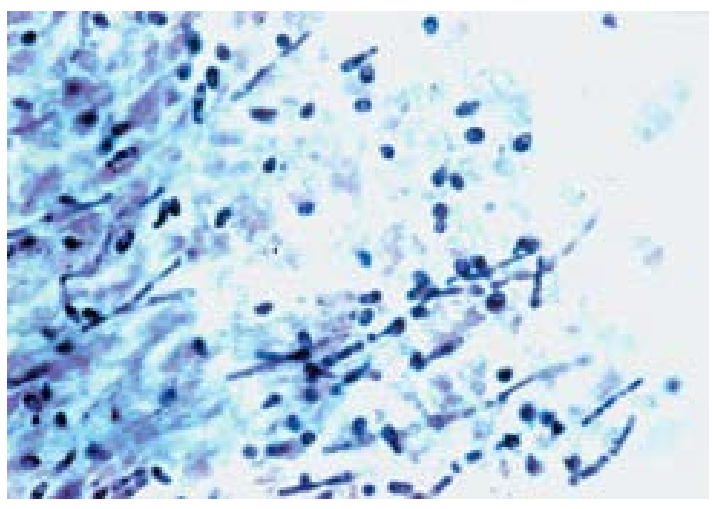

Figure 5. Wet mount from white esophageal plaques detects pseudohyphae and budding yeast cells indicating esophageal candidiasis (gram stain) [14].

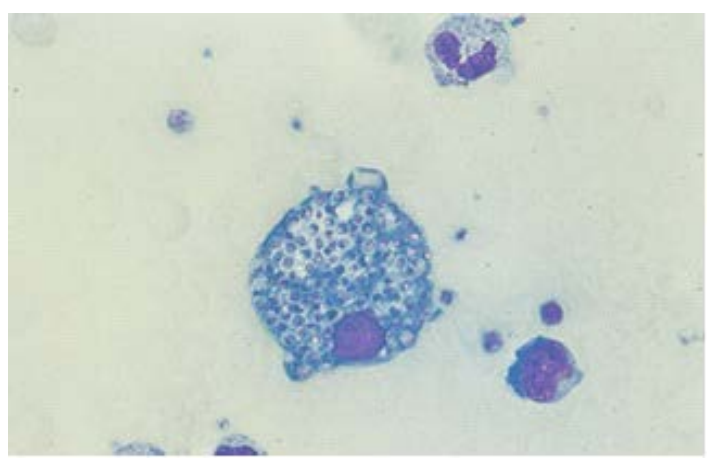

Figure 6. Bone marrow aspirate stained with Giemsa showing the yeast cells of Histoplasma capsulatum inside the monocytes [12].

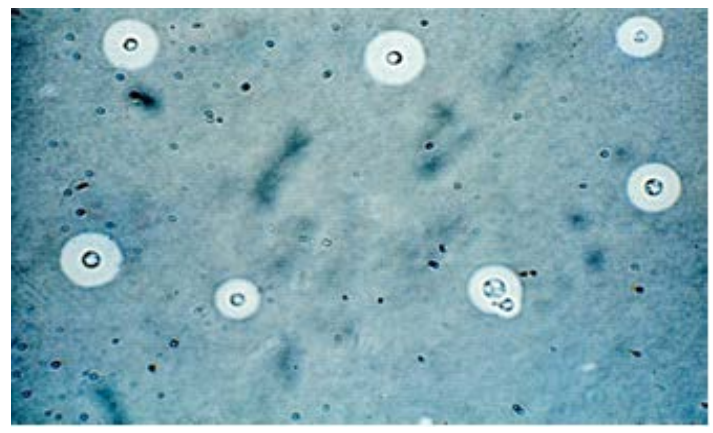

Figure 7. India ink preparation showing Cryptococcus neoformans in CSF [15].

\section{Culture and identification}

There are many primary media which are used for fungal element isolation such as Sabouraud dextrose agar (SDA), inhibitory mold agar (IMA)', mycobiotic agar, brain heart infusion agar (BHI), and Trichophyton agars. Media for primary isolation of fungi should be general purpose, and able to support the growth of most pathogenic fungi. The growth of bacteria or saprophytic fungi can be reduced by addition of inhibiting agents. Different blood culture systems are used for blood and bone marrow specimens, and then subcultures on appro- 
priate media could be performed [17].

Molds are incubated at room temperature $\left(22^{\circ} \mathrm{C}\right.$ to $\left.25^{\circ} \mathrm{C}\right)$, and lyeasts are incubated at $28^{\circ} \mathrm{C}$ to $37^{\circ} \mathrm{C}$. ¡All fungal cultures should be observed on 1 st and 2 nd day after inoculation then every other day for one week, then every 5 - 6 days until 4 - 6 weeks [8]?

Identification of species can be done by using conventional methods which are based on: 1) colony morphology including color, texture, and reverse side appearance, 2) microscopic examination that reveals cells morphology either yeast or hyphae, macroconidia, microconidia, phialides, septation and chlamydospore, and 3) biochemical features such as sugar assimilation, and urease test [18].

Cultivation on morphology agars provides recognition of four different types of yeast growth morphology: blastoconidia, chlamydoconidia, pseudohyphae and arthroconidia after $72 \mathrm{~h}$. Germ tube production test is used for identification of germ tube positive yeasts such as Candida albicans after $3 \mathrm{~h}$. Chromogenic agars incorporate multiple chemical dyes in a solid medium that allow rapid identification lof Candida species after $24 \mathrm{~h}$ (Figure 8) [19].

Api ID 20C, and 32C are commercial products based on biochemical reactions of yeast isolates such as carbohydrate assimilation, and urease production within 48 to 72 hours. These reactions could be identified visually or by using mini API system. VITEK 2 system is a fully automated system for identification and susceptibility testing of microorganisms?. VITEK ID-YST cardis allow for identification of yeast and yeast like organisms in $15 \mathrm{hrs}$ [20].

\section{Serological Diagnosis of Fungal Infections}

Antigen detection can be used to screen high-risk patients to invasive fungal infections, facilitate early diagnosis, and also to monitor the response to therapy. Antibody titer is often used in diagnosing endemic mycoses, which are difficult to be detected by traditional methods.| However, detection of fungal antibodies in serum is of limited value for immunocompromised patients. Combination of

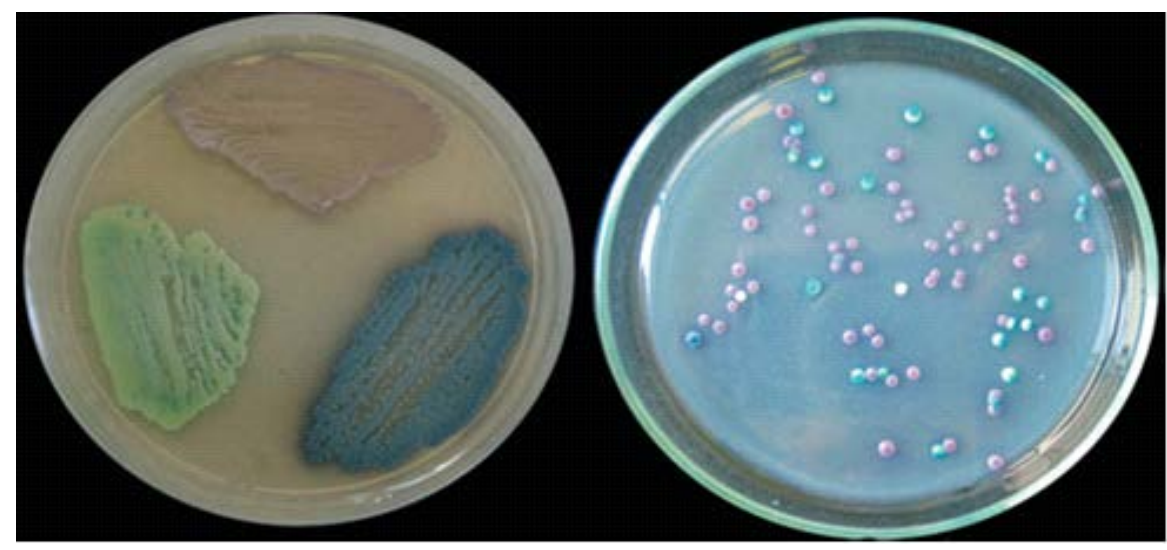

(a)

(b)

Figure 8. Chromagar plates showing Candida growth. C. albicans, C. tropicalis, and C. glabrata appear as green, blue, and purple colonies, respectively. (a) cultures from reference strains; (b) clinical sample from a patient with denture stomatitis oral [20]. 
antigen and antibody tests improves both specificity and sensitivity [21].

Mannan detection in serum and other body fluids can be performed for diagnosis of invasive candidiasis by many serological reactions like enzyme linked immunosorbent àssay (ELISA), or radioimmunoassay (RIA). Anti-mannan antibodies are found in patients either colonized or infected so, rising titer has to be observed in serial serum samples.' Currently, they are detected by ELISA assay, and if it is used together with mannan assay, their sensitivity and specificity for [diagnosing IC improves significantly [22]?

Latex agglutination (LA) assay" is the most often assay used for Cryptococcal polysaccharide antigen detection in serum and CSF specimens.'? Cryptococcal antigen assay by EIA format is commonly used. Lateral flow immune-chromatographic assay (LFA), for rapid 20 minutes. Semi quantitative detection of cryptococcal antigen in iserum, urine, or CSF has been developed. LFA is reportedly more rapid and convenient than standard latex agglutination (LA) assay [23].'Detection of circulating Galactomannan (GM) by ELISA lassay in serum and bronchoalveolar lavage (BAL) fluid, is generally used for diagnosis of invasive aspergillosis [21]?

A promising diagnostic method which is based on the detection of Aspergillus-specific JF5 antigen had been developed. The JF5 is an extracellular glycoprotein that is exclusively secreted during lactive growth of the fungus and represents a surrogate marker of ¡Aspergillus infection.» This test can overcome the cross-reactivity or a false-positive |results which loccur with circulating galactomannan detection by ELISA assay [24]?

Histoplasma polysaccharide antigen can be detected by lquantitative enzyme immunoassay even at early stages of disease. It can be applied on serum, urine, CSF, and BAL fluid samples; [25]?

11-3-beta-D-glucan is a cell wall component of most fungi except Cryptococcus and Zygomycetes. Recently, it is introduced as a reliable marker of an invasive fungal infection, and has therefore been added to the European Organization for Research and Treatment bof Cancer/Mycoses Study Group (EORTC/MSG) criteria as a marker for invasive fungal diseases. Fungi tell assay, which is an ELISA technique, is widely used to detect jerum BG concentrations as low as $1 \mathrm{pg} / \mathrm{mL}$. False-positive reactions have been reported in patients with bacterial infections, in those who have recently received albumin or immunoglobulin products. Studies suggested that raising the cut-off level of can improve the specificity of the test, without loss of sensitivity [26] [27].

\section{Antifungal Susceptibility Testing (AST)}

The national committee for clinical laboratory standards introduce (NCCLS M27-A documents and M38-A) document for antibiotic susceptibility testing of yeasts, and molds respectively. They are either macrodilution or microdilution format. They specify inoculum size, test medium, incubation time for some antifungal drugs [28] [29].

Agar based methods as disk diffusion test and E test provide simple, rapid, 
and inexpensive methods for drug susceptibility testing. M44-A is a recent reference method for antifungal disk diffusion with reading after $24 \mathrm{~h}$ [30].

Automated systems for antifungal susceptibility testing have been recently introduced to save time and provide more accurate results. Sensititre system provides clearer visual end points and acceptable minimum inhibitory concentrations (MICs) as those bobtained using the standard broth dilution method. VITEK2 is a fully automated and standardized system which used for antifungal susceptibility testing of fungi especially candida species. It utilizes closed dehydrated drug cards from which MICs are automatically determined after $13 \mathrm{~h}$ [31]. Flowcytometry (FCM) assay for AST is also available for Candida and Aspergillus species, and provides rapid $(4 \mathrm{~h})$ results [32].

\section{Molecular approaches for diagnosis of fungal infections}

Molecular techniques" are rapid, less invasive, and provides reliable species identification. They can be applied directly on clinical specimens?'

Fluorescent in situ Hybridization (FISH) is a powerful probe based method for in situ detection of active growing lorganisms in environmental samples. It can visualize the exact location of particular DNA or RNA sequences in the cytoplasm,' 'organelles, or nuclei of biological materials". Peptide nucleic acid (PNA) probes allow for identification of candida species from positive blood cultures in less than $3 \mathrm{~h} \mathrm{[33].}$

The major step of FISH involves the preparation of biological materials or environmental samples, and the labelling (incorporation of a fluorescent label/marker) of a nucleic acid sequence to form a probe. Then, under controlled experimental conditions, the probe is hybridized to the DNA or RNA in biological materials to form a double-stranded molecule. Finally, the sites of hybridization are detected and visualized [33].

Microarray is another probe based method. It allows thousands of probeto-ttarget sequence binding reactions to occur on the surface of a tiny microchip, and then are detected and analyzed by computer [34]. With amplification based methods, PCR as little as a few picograms of input DNA can be amplified so that after 30 - 40 cycles, the resulting product can be visualized on an agarose gel or detected using chemiluminescence, spectrophotometry, or a laser instrument [35].

Real time PCR combines PCR, probe hybridization, and signal generation in one step. The Taq Man probe consists of a reporter dye with a fluorescein derivative at the $5^{\prime}$ end and a 3 ' quencher dye. The probe is cleaved by the $55^{\prime \prime}$ endonuclease activity of Taq polymerase, thus releasing the reporter dye fluorescence only when it is hybridized to a complementary target. The amount of reporter dye released is proportional to the amount of DNA amplified by PCR [36].

Multiplex PCR assay can detect a wide variety of fungi simultaneously in the same specimen, which is time-saving and more cost-effective than conventional PCR. The platform consists of two rounds of amplification. In the first step, a multiplex PCR is performed at 10 to 15 cycles to allow enrichment of target DNA without creating competition between amplicons [37]. 
This product is diluted and used as template for the second amplification that consists of multiple individual quantitative PCR reactions with primers nested within those used in the multiplex PCR. Up to 72 different PCR reactions can be multiplexed and performed simultaneously. Fluorescence is measured by SYBR green technology at the end of each extension cycle, and melt-curve analysis provides species-specific or gene-specific identification [37].

The incorporation of two sets of species-specific primers for each target ensures correct amplification and detection, thus avoiding the expense of DNA probes. SYBR green detection also increases the multiplexing and quantitative capacity of real-time PCR systems, which are usually limited by the availability of fluorescent channels and the need to optimize each individual multiplex PCR [37].

Light Cycler Septi Fast (LC-SF) technology for pathogen in patients with sepsis, is a new commercial multiplex PCR which is able to detect many pathogens responsible for bloodstream infections within hours. It is able to identify five common Candida species (C. albicans, C. tropicalis, C. parapsilosis, C. krusei, $C$. (glabrata) as well as Aspergillus fumigatus by using probes for PCR that target the internal transcribed spacer (ITS) between $18 \mathrm{~S}$ and $5.6 \mathrm{~S}$ fungal ribosomal RNA |(rRNA) [38].

Another recent development is the multianalyte profiling (xMAP) system. This assay utilizes a novel flow cytometer and tiny beads color-coded with" unique dyes that enable each bead to be distinguished from all other beads in the laser reader.

Loop mediated isothermal amplification is the amplification of few copies of target DNA under isothermal conditions It can be used for the rapid detection lof pathogenic fungi like Penicillium marneffei, and Cryptococcus species. It is rapid and simple amplification method which can be used in developing countries without requiring sophisticated equipment or skilled personnel. In PCR ELISA test PCR amplification, hybridization with the complementary labeled probe, and detection of reaction products by EIA is performed. It can identify different dermatophytes species. It has a greater sensitivity compared with dermatophyte cultures [39].

Matrix-assisted laser desorption/ionization-time of flight mass ispectrometry (MALDI-TOF MS)

MALDI-TOF MS is one of the proteomic approaches that made revolutionary changes in clinical microbiology laboratories all over the world." MALDITOF fingerprinting is currently emerging as a rapid and reliable tool for identifying yeasts and molds. The obtained mass spectral finger prints act as relative unique signatures for each microorganism and thus ideal for accurate identification at species levels [40].

Currently, there are four commercial systems available: the MALDI Biotyper (Bruker Daltonics, Bremen, Germany), the AXIMA SARAMIS database (Anagnos Tec, Potsdam, Germany and Shimadzu, Duisburg, Germany), and lately the Andromas (Andromas, Paris, France) and VITEK MS systems (bio Merieux, Marcy l'Etoile, France) However, this method has not yet been comparatively 
evaluated in a large number of clinical isolates concomitantly with accepted standards [41].

In a study by Lacroix et al., [42], the identification results by MALDI-TOF MS were concordant with those of the conventional culture-based method for 95.9\% of Pandida albicans and $86.5 \%$ of non albicans Candida species. Results were available in $30 \mathrm{~min}$, suggesting that this approach is a reliable, time saving tool for routine identification of Candida species which cause bloodstream infection. Far less data are available for the differentiation of molds like AspergilIus," Fusarium, and dermatophytes."

\section{Nanotechnology approaches for diagnosis of fungal infections}

Metallic nanoparticles as gold or silver nanoparticles have many electronic, optical and chemical properties. For example, gold nanoparticles conjugated with specific oligonucleotides can be detected by color changes.

T2 magnetic resonance (T2MR)-based biosensing has been used for the detection of a wide range of target types, including proteins, drugs, pathogens, enzymes, and cells, in a variety of complex matrices, such as culture media, cell lysate, urine, serum, plasma, sputum, fine-needle aspirates, and blood [43]. An automated T2 Candida assay is developed as anid diagnostic test for candidemia using T2 magnetic resonance technology, which is a combination of nanoparticle-based hybridization and PCR amplification [43].

T2MR can be used for detection of nucleic acids by synthesizing a mixture of two populations of superparamagnetic particles, each functionalized with different oligonucleotide capture probes: one conjugated to a capture probe hybridizing to the 5 end of the single stranded DNA target and one hybridizing to the 3 end of the DNA [43].

This technique is able to differentiate Candida species including C. albicans, C. Ptropicalis, C. krusei, C. glabrata, and C. parapsilosis directly from whole" blood. T2MR also provides faster time to results compared with FISH bor MALDI TOF. In addition, it yields with $98 \%$ positive and $100 \%$ negative agreement compares with blood culture results in clinical studies [43].

\section{Conclusions}

- Traditional methods such as wet mount examination and microbiological cultures remain the gold standard for fungal disease diagnosis.

- Antigen detection tests can be used for monitoring the response to antifungal therapy and screening immunocompromised individuals for IFI.

- Combination of $\mathrm{Ag} / \mathrm{Ab}$ increases the specificity and sensitivity of both tests and provides more accurate results.

- VITEK 2 and flow cytometry are reliable and rapid systems for antifungal susceptibility tests.

- Molecular techniques, MALDI-TOF MS, and nanotechnology provide early and accurate identification of fungi, thereby enhancing epidemiological studies as well as the diagnosis. Challenges remain to determine sensitivity, specificity, accuracy and cost. 


\section{References}

[1] Vandeputte, P., Ferrari, S. and Coste, A.T. (2012) Antifungal Resistance and New Strategies to Control Fungal Infections. International Journal of Microbiology, 2012, Article ID: 713687. https://doi.org/10.1155/2012/713687

[2] Low, C.Y. and Rotstein, C. (2011) Emerging Fungal Infections in Immunocompromised Patients. F1000 Medicine Reports, 3, 14. https://doi.org/10.3410/M3-14

[3] Jain, A., Jain, S. and Rawat, S. (2010) Emerging Fungal Infections among Children: A Review on Its Clinical Manifestations, Diagnosis, and Prevention. Journal of Pharmacy and Bioallied Sciences, 2, 314-320. https://doi.org/10.4103/0975-7406.72131

[4] Nenoff, P., Krüger, C., Schaller, J., et al. (2014) Mycology-An Update Part 2: Dermatomycoses: Clinical Picture and Diagnostics. Journal der Deutschen Dermatologischen Gesellschaft, 12, 749-777.

[5] Bustamante, B. and Campos, P.E. (2011) Mycoses Involving Skin and Subcutaneous Tissues, Eumycetoma. In: Kauffman, C.A., Pappas, P.G., Sobel, J.D. and Ismukes, W.D., Eds., Essentials of Clinical Mycology, Springer, New York, 415-425.

[6] Willinger, B., Kienzl, D. and Kurzai, O. (2014) Diagnostics of Fungal Infections. In: Esser, K. and Kurzai, O., Eds., The Mycota, Springer, Heidelberg, New York, 229263. https://doi.org/10.1007/978-3-642-39432-4_13

[7] Posteraro, B., Torelli, R., Carolis, E.D., et al. (2011) Update on the Laboratory Diagnosis of Invasive Fungal Infections Mediterranean. Journal of Hematology and Infectious Diseases, 3, e2011002. https://doi.org/10.4084/mjhid.2011.002

[8] Nye, M.B., Beard, M.A. and Body, B.A. (2006) Diagnostic Mycology: Controversies and Consensus-What Should Laboratories Do? Part I. Clinical Microbiology Newsletter, 28, 121-127. https://doi.org/10.1016/j.clinmicnews.2006.08.001

[9] Reiss, E., Shadomy, H.J. and Lyon, G.M. (2011) Introduction to Fundamental Medical Mycology. In: Reiss, E., Shadomy, H.J. and Lyon, G.M., Eds., Fundamental Medical Mycology, John Wiley \& Sons, Hoboken, 3-28.

https://doi.org/10.1002/9781118101773.ch1

[10] Vyzantiadis, T., Johnson, E. and Kibbler, C. (2012) From the Patient to the Clinical Mycology Laboratory: How Can We Optimise Microscopy and Culture Methods for Mould Identification? Journal of Clinical Pathology, 65, 475-483. https://doi.org/10.1136/jclinpath-2011-200500

[11] Stevens, D.A. (2002) Diagnosis of Fungal Infections: Current Status. Journal of Antimicrobial Chemotherapy, 49, 11-19. https://doi.org/10.1093/jac/49.suppl_1.11

[12] Fothergill, A.W. (2014) Medically Significant Fungi. In: Mahon, C.R., Lehman, D.C. and Manuselis, G., Eds., Textbook of Diagnostic Microbiology, Elsevier Saunders, Missouri, 589-623.

[13] Moore, C.B. and Richardson, M.D. (2014) Infections Caused by Mucorales. In: Esser, K. and Kurzai, O., Eds., The Mycota, Springer, Heidelberg, New York, 205-229. https://doi.org/10.1007/978-3-642-39432-4_12

[14] Kauffman, C.A. (2007) Introduction to the Fungi and Mycoses. In: Engleberg, N.C., DiRita, V. and Dermody, T.S., Eds., Schaechter's Mechanism of Microbial Disease, Lippincott Williams and Wilkins, Baltimore, 468-472.

[15] Baddley, J.W. and Dismukes, W.E. (2011) Cryptococcosis. In: Kauffman, C.A., Pappas, P.G., Sobel, J.D. and Dismukers, W.E., Eds., Essentials of Clinical Mycology, Springer, New York, 207-226. https://doi.org/10.1007/978-1-4419-6640-7_12

[16] Ahmad, I. and Khan, M. (2012) Microscopy in Mycological Research with Especial Reference to Ultrastructures and Biofilm Studies. Current Microscopy Contribu- 
tions to Advances in Science and Technology. Formatex Research Center, Badajoz, 646-659.

[17] Mulla, A.F., Shah, A.A., Koshy, A.V., et al. (2015) Laboratory Diagnosis of Fungal Infection. Universal Research Journal of Dentistry, 5, 49-53.

https://doi.org/10.4103/2249-9725.157723

[18] Cornely, O.A., Bassetti, M., Calandra, T., et al. (2012) ESCMID Guideline for the Diagnosis and Management of Candida Diseases 2012: Non-Neutropenic Adult Patients. Clinical Microbiology and Infection, 18, 19-37. https://doi.org/10.1111/1469-0691.12039

[19] Fenn, J. (2007) Update of Medically Important Yeasts and a Practical Approach to Their Identification. Laboratory Medicine, 38, 178-183. https://doi.org/10.1309/WFJEBPMDHV4EAT0J

[20] Neppelenbroek, K.H, Seó, R.S., Urban, V.M., et al. (2013) Identification of Candida Species in the Clinical Laboratory: A Review of Conventional, Commercial, and Molecular Techniques. Oral Diseases, 20, 329-344.

https://doi.org/10.1111/odi.12123

[21] Pemán, J. and Zaragoza, R. (2012) Combined Use of Nonculture-Based Lab Techniques in the Diagnosis and Management of Critically Ill Patients with Invasive Fungal Infections. Expert Review of Anti-Infective Therapy, 10, 1321-1330. https://doi.org/10.1586/eri.12.128

[22] Deorukhkar, S.C. and Saini, S. (2014) Laboratory Approach for Diagnosis of Candidiasis through Ages. International Journal of Current Microbiology and Applied Sciences, 3, 206-218.

[23] McMullan, B.J., Halliday, C., Sorrell, T.C., et al. (2012) Clinical Utility of the Cryptococcal Antigen Lateral Flow Assay in a Diagnostic Mycology Laboratory. PLoS ONE, 7, e49541. https://doi.org/10.1371/journal.pone.0049541

[24] Lackner, M. and Lass-Florl, C. (2013) Up-Date on Diagnostic Strategies of Invasive Aspergillosis. Current Pharmaceutical Design, 19, 3595-3614.

https://doi.org/10.2174/13816128113199990323

[25] Arango-Bustamante, K., Restrepo, A., Cano, L.E., et al. (2013) Diagnostic Value of Culture and Serological Tests in the Diagnosis of Histoplasmosis in HIV and NonHIV Colombian Patients. American Journal of Tropical Medicine and Hygiene, 89, 937-942. https://doi.org/10.4269/ajtmh.13-0117

[26] Khodadadi, H., Mirhendi, H., Makimuraet, K., et al. (2014) $\beta$-D-Glucan Assay in Diagnosis and Monitoring the Systemic Candidiasis in a Rat Model. Jundishapur Journal of Microbiology, 7, e10247. https://doi.org/10.5812/jjm.10247

[27] Hammarström, H., Kondori, N., Friman, V. and Wennerås, C. (2015) How to Interpret Serum Levels of Beta-Glucan for the Diagnosis of Invasive Fungal Infections in Adult High-Risk Hematology Patients: Optimal Cut-Off Levels and Confounding Factors. European Journal of Clinical Microbiology \& Infectious Diseases, 34, $917-$ 925. https://doi.org/10.1007/s10096-014-2302-9

[28] National Committee for Clinical Laboratory Standards (1997) Reference Method for Broth Dilution Antifungal Susceptibility Testing of Yeasts. Approved Standard. NCCLS Document M27-A, National Committee for Clinical Laboratory Standards, Wayne.

[29] National Committee for Clinical Laboratory Standards (2002) Reference Method for Broth Dilution Antifungal Susceptibility Testing of Filamentous Fungi. Approved Standard. NCCLS Document M38-A, National Committee for Clinical Laboratory Standards, Wayne. 
[30] National Committee for Clinical Laboratory Standards (2004) Method for Antifungal Disk Diffusion Susceptibility Testing of Yeasts: Approved Guideline. NCCLS document M44-A, National Committee for Clinical Laboratory Standards, Wayne.

[31] Astvad, K.M., Perlin, D.S., Johansen, H.K., Perlin, D.S., Johansen, H.K., Jensen, R.H. and Arendrup, M.C. (2012) Evaluation of Caspofungin Susceptibility Testing by the New Vitek 2 AST-YS06 Yeast Card Using a Unique Collection of FKS Wild-Type and Hot Spot Mutant Isolates, Including the Five Most Common Candida Species. Antimicrobial Agents and Chemotherapy, 57, 177-182.

https://doi.org/10.1128/AAC.01382-12

[32] Chaturvedi, V., Ramani, R. and Pfaller, M.A. (2004) Collaborative Study of the NCCLS and Flow Cytometry Methods for Antifungal Susceptibility Testing of Candida Albicans. Journal of Clinical Microbiology, 42, 2249-2251. https://doi.org/10.1128/JCM.42.5.2249-2251.2004

[33] Alexander, B.D. and Pfaller, M.A. (2006) Contemporary Tools for the Diagnosis and Management of Invasive Mycoses. Clinical Infectious Diseases, 43, S15-S27. https://doi.org/10.1086/504491

[34] Tsui, C.K., Woodhall, J., Chen, W., et al. (2011) Molecular Techniques for Pathogen Identification and Fungus Detection in the Environment. Imafungus, 2, 177-189. https://doi.org/10.5598/imafungus.2011.02.02.09

[35] Arunmozhi, B.S., Sigler, L. and Brandt, M.E. (2007) DNA and the Classical Way: Identification of Medically Important Molds in the 21st Century. Medical Mycology, 45, 475-490. https://doi.org/10.1080/13693780701449425

[36] Qishui, O., Ling, J., Ni, L., Yang, B. and Li, W. (2011) Comparison of Real-Time Florescence Quantitative PCR Measurements of VAD1 mRNA with Three Conventional Methods in Diagnosis and Follow-Up Treatment of Cryptococcus Neoformans Infection. Mycoses, 55, 326-332. https://doi.org/10.1111/j.1439-0507.2011.02100.x

[37] Zhang, S.X. (2013) Enhancing Molecular Approaches for Diagnosis of Fungal Infections. Future Microbiology, 8, 1599-1611. https://doi.org/10.2217/fmb.13.120

[38] Ortiz Ibarra, J., Valdez, P.T., Mendez, E.V., et al. (2015) Evaluation of the LightCycler SeptiFast Test in Newborns With Suspicion of Nosocomial Sepsis. Iranian Journal of Pediatrics, 25, e253. https://doi.org/10.5812/ijp.253

[39] Vanichanan, J. and Ostrosky-Zeichner, L. (2015) Molecular Diagnosis in Fungal Infection Control. Current Treatment Options in Infectious Diseases, 7, 1-13. https://doi.org/10.1007/s40506-015-0040-x

[40] Brun, S., Madrid, H., Van Den Ende, G.B., et al. (2013) Multilocus Phylogeny and MALDI-TOF Analysis of the Plant Pathogenic Species Alternariadauci and Relatives. Fungal Biology, 117, 32-40. https://doi.org/10.1016/j.funbio.2012.11.003

[41] Bader, O. (2013) MALDI-TOF-MS-Based Species Identification and Typing Approaches in Medical Mycology. Proteomics, 13, 788-799.

https://doi.org/10.1002/pmic.201200468

[42] Lacroix, C., Gicquel, A., Sendid, B., et al. (2014) Evaluation of Two Matrix-Assisted Laser Desorption Ionization-Time of Flight Mass Spectrometry (MALDI-TOF MS) Systems for the Identification of Candida Species. Clinical Microbiology and Infection, 20, 153-158. https://doi.org/10.1111/1469-0691.12210

[43] Neely, L.A., Audeh, M., Phung, N.A., et al. (2013) T2 Magnetic Resonance Enables Nanoparticle-Mediated Rapid Detection of Candidemia in Whole Blood. Science Translational Medicine, 5, 182 ra54. 
Submit or recommend next manuscript to SCIRP and we will provide best service for you:

Accepting pre-submission inquiries through Email, Facebook, LinkedIn, Twitter, etc. A wide selection of journals (inclusive of 9 subjects, more than 200 journals)

Providing 24-hour high-quality service

User-friendly online submission system

Fair and swift peer-review system

Efficient typesetting and proofreading procedure

Display of the result of downloads and visits, as well as the number of cited articles Maximum dissemination of your research work

Submit your manuscript at: http://papersubmission.scirp.org/

Or contact ojmm@scirp.org 